\title{
Development of a Monochromated and Aberration-Corrected Low-Voltage (S)TEM
}

\author{
Masaki Mukai ${ }^{1}$, Shigeyuki Morishita ${ }^{1}$, Atsushi Kimura ${ }^{1}$, Akihiro Ikeda ${ }^{1}$, Kazunori Somehara ${ }^{1}$, \\ Hidetaka Sawada ${ }^{1}$, Luiz H. G. Tizei ${ }^{2}$, Yung-Chang Lin $^{2}$, Koji Kimoto ${ }^{3}$ and Kazu Suenaga ${ }^{2}$ \\ 1. JEOL Ltd., 3-1-2 Musashino, Akishima, Tokyo 196-8558, Japan \\ 2. Nanotube Research Center, National Institute of Advanced Industrial Science and Technology (AIST), \\ Central 5, 1-1-1 Higashi, Tsukuba, Ibaraki 305-8565, Japan \\ 3. National Institute for Materials Science (NIMS), 1-1, Namiki, Tsukuba, Ibaraki, 305-0044, Japan
}

Low-voltage scanning/transmission electron microscope (STEM/TEM) equipped with delta-type aberration correctors [1] was developed under a project "Triple-C phase-1" to observe the atomic structure of carbon materials with less knock-on damage. This microscope enables us to observe and analyze defected structures of graphene edge by using electron energy-loss spectroscopy (EELS) at an atomic scale [2]. However this microscope was equipped with a cold field emission gun to obtain high brightness therefore its energy resolution remains to be approximately $0.3 \mathrm{eV}$. To study the electronic structures of materials in detail, we have developed a monochromator working at $15-60 \mathrm{kV}$ for a lowvoltage aberration-corrected microscope under a project “Triple-C phase-2”, whose targeted energy resolution is better than $25 \mathrm{meV}$.

The developed microscope, which is based on JEM-ARM200F, is equipped with a high resolution EELS (Quantum-ERS optimized in low-voltage, GATAN Inc.) and delta-type aberration correctors for STEM and TEM. The optical system for the monochromator is chosen to be a double Wien-filter. The Wienfilter arranged between the extraction anode of Schottky source and the accelerator, as we developed in the previous design [3]. After the monochromator, the electron probe is achromatic and the energy spread is controlled by the width of the slit, independently on the probe size at the specimen. In addition, the setting of the monochromator and the electron trajectories inside the monochromator are independent of the change of the accelerating voltage, since the monochromator is located before the accelerator and the potential along the optical axis inside the monochromator is kept constant.

The ultimate energy resolution with acquisition time of $2 \mathrm{~ms}$ was obtained to be $14 \mathrm{meV}$ with a $0.1 \mu \mathrm{m}$ width slit at $30 \mathrm{kV}$ as shown in Fig.1 (a). Figure 1(b) shows the low loss spectrum from Hexagonal BN obtained with an energy resolution of $22 \mathrm{meV}$ and an acquisition time of $300 \mathrm{~ms}$ at $30 \mathrm{kV}$. This spectrum, which was measured with a probe size of about $1 \mathrm{~nm}$, a beam current of about $10 \mathrm{pA}$, a convergence semi-angle of $15 \mathrm{mrad}$ and a collection semi-angle of $30 \mathrm{mrad}$, showed a sharp peak corresponding to an optical phonon at about $170 \mathrm{meV}$. These results suggest that the microscope enables us to analyze materials with very high energy resolution $\leq 25 \mathrm{meV}$ in nanometer scale, owing to a large scattering cross-section and small specimen damage by using lower voltage electrons.

Figure 2 shows a monochromated and aberration-corrected TEM image of single-layered graphene obtained with an energy spread of $172 \mathrm{meV}$ at $60 \mathrm{kV}$. This image implies that the monochromated electron source in aberration-corrected TEM provides clearly resolved C-C bonds and the enhancement of spatial resolution arising from a small chromatic aberration in TEM at low accelerating voltage.

This work is supported by Japan Science and Technology agency, Research Acceleration Program.

\section{References}

[1] H. Sawada, et al.: J. Electron. Microsc. 58 (2009) 341.

[2] K. Suenaga and M. Koshino, Nature 468 (2010) 1088.

[3] M. Mukai, et al.: Ultramicroscopy 140 (2014) 37. 

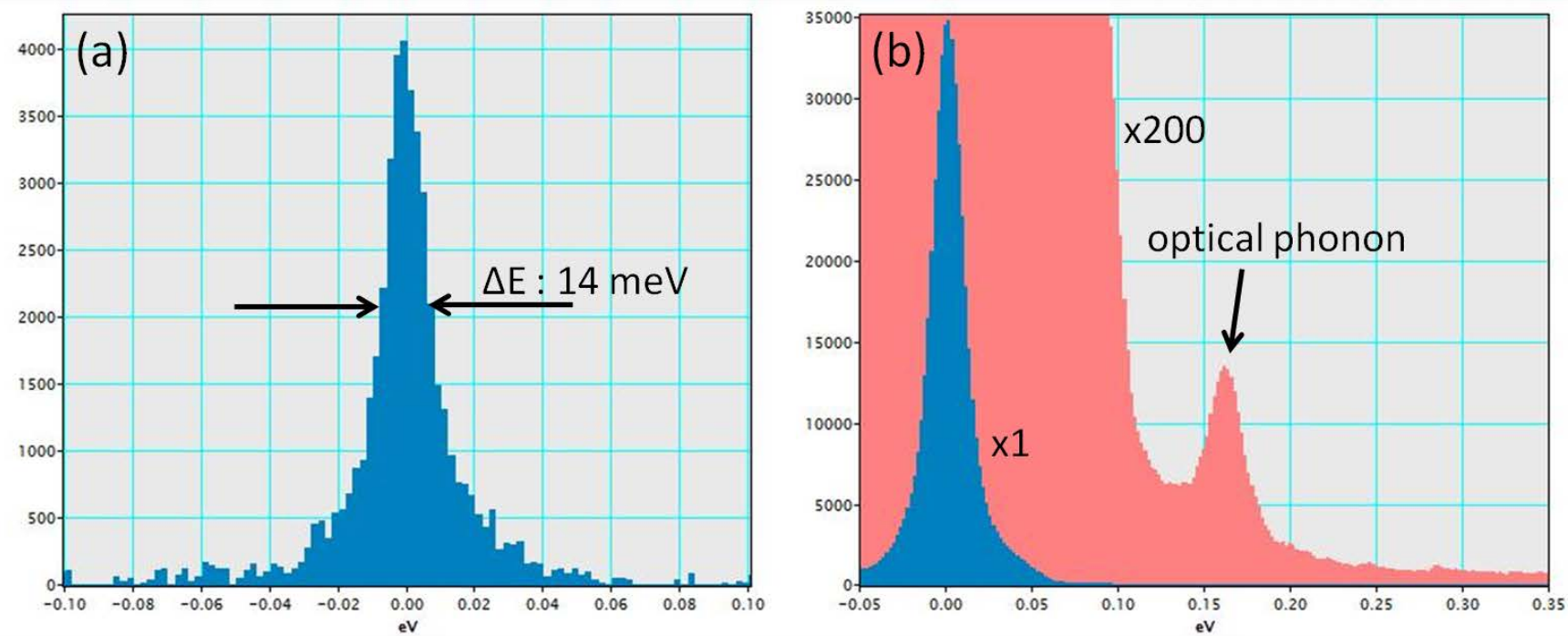

Fig. 1. (a) Intensity profile of the zero-loss peak with the energy resolution of $14 \mathrm{meV}$, recorded using a slit of $0.1 \mu \mathrm{m}$ wide and an acquisition time of $2 \mathrm{~ms}$ at $30 \mathrm{kV}$. (b) Low loss spectrum and its magnified spectrum from hexagonal BN obtained with an energy resolution of $22 \mathrm{meV}$ and an acquisition time of $300 \mathrm{~ms}$ at $30 \mathrm{kV}$, showing a signal at $\fallingdotseq 170 \mathrm{meV}$ as indicated by an arrow.

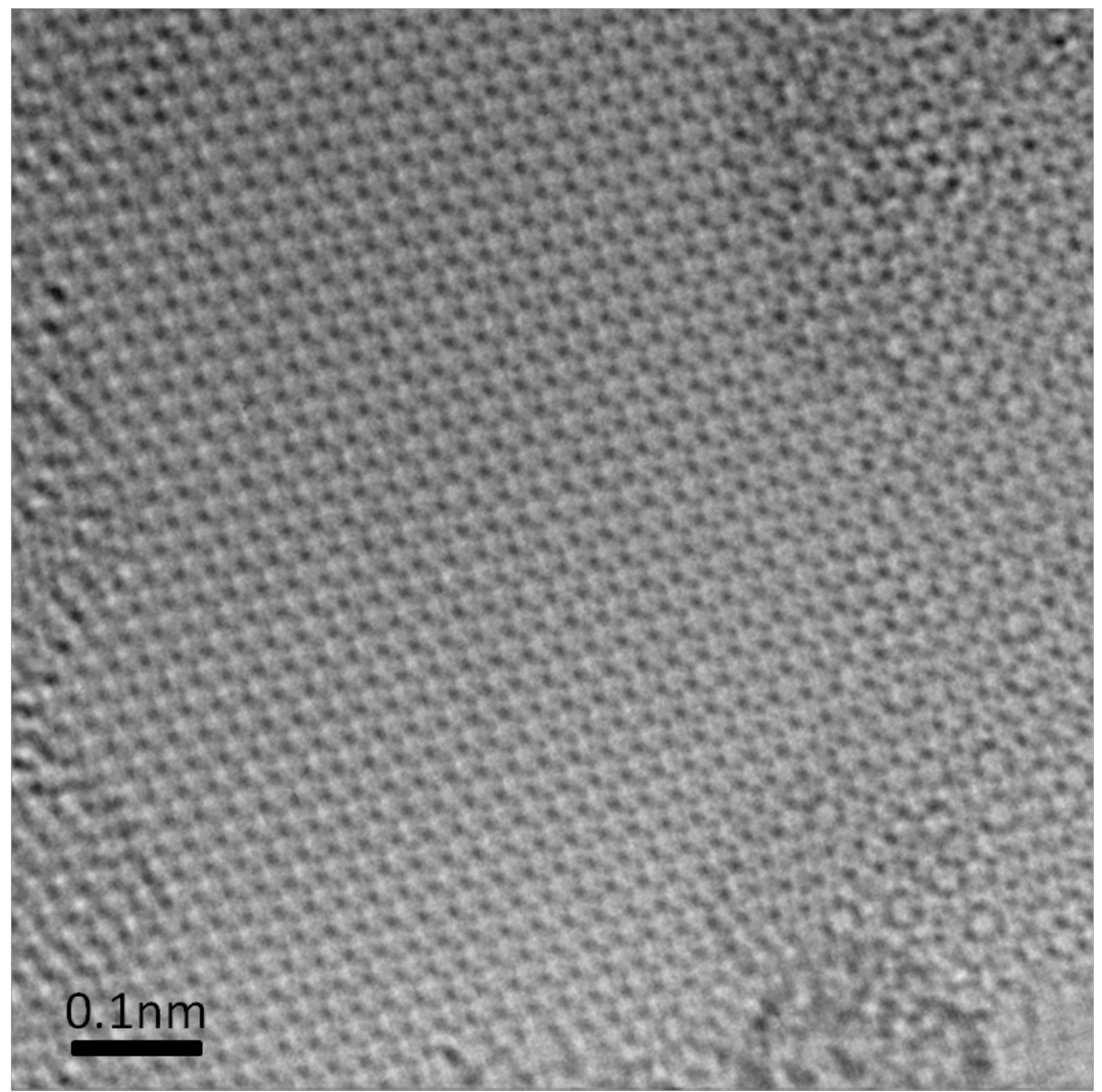

Fig. 2. TEM image of single-layered graphene obtained with an energy spread of $172 \mathrm{meV}$ at $60 \mathrm{kV}$ by the monochromated and aberration-corrected microscope. 